\title{
POLYPOID NASAL MASSES; SURGICAL PATHOLOGY
}

\author{
1. MBBS, FCPS. \\ Assistant Professor, \\ Department of Pathology. \\ Jinnah Medical College, Peshawar. \\ 2. MBBS, M. Phil, PhD. \\ Professor, \\ Department of Pathology, \\ Jinnah Medical College, Peshawar \\ 3. MBBS, FCPS, FRCS \\ Professor, \\ Department of Surgery. \\ Jinnah Medical College, Peshawar \\ 4. MBBS, MPhil \\ Professor, \\ Department of Physiology, \\ Jinnah Medical College, Peshawar.
}

Correspondence Address:

Dr. Amjad Ali Khan

Assistant Professor,

Department of Pathology,

Jinnah Medical College, Peshawar.

amjadalikhan52@hotmail.com

Article received on:

28/02/2017

Accepted for publication:

$15 / 09 / 2017$

Received after proof reading:

03/11/2017
Amjad Ali Khan ${ }^{1}$, Abdul Shaheed Asghar ${ }^{2}$, Muhammad Ishaq ${ }^{3}$, Israr Ahmed Akhund ${ }^{4}$

ABSTRACT... Objectives: The purpose of this study is firstly to signify the occurrence of unusual nasal polypoid masses by analyzing their histopathological spectrum and to be on the lookout for them. Secondly to highlight the fact that nasal polypoid masses should not be taken lightly as they may be representing an underlying grave pathological process meriting urgent medical advice. Study Design: Retrospective/observational study. Setting: Charsada Teaching Hospital affiliated with Jinnah Medical College Peshawar. Period: March 2010 to March 2015. Methods: In this study, all the surgical pathology cases with clinical diagnosis of nasal polyps with no suspicion for malignancy were retrieved from archives of Charsada Teaching Hospital affiliated with Jinnah Medical College Peshawar. The slides and diagnoses of all the retrieved cases were reviewed. The number of cases for each diagnostic category were recorded and analyzed according to the non-neoplastic, neoplastic and their subcategories. Results: The review of cases from March 2010- March 2015 showed that most of the polypoid masses were non-neoplastic, while only few were neoplastic. Of all the non-neoplastic masses, inflammatory/ allergic polyps were much more common followed bypolyps with fungal infections and eosinophilic angiocentric fibrosis. Amongst the neoplastic masses, benign tumors were more common than the malignant. The benign masses comprised of hemangioma, angiofibroma, inverted papilloma and schwannoma. The malignant nasal masses constituted one case each of polypoid extraskeletal Ewings sacoma, plasmacytoma, non-Hodgkin lymphoma and renal cell carcinoma. Conclusions: Awareness about the occurrence of the usual and unusual entities in the nasal cavities needs to be increased. Important diagnostic categories requiring urgent management include polyps with invasive fungal infection, inverted papilloma and all malignant cases. Therefore, all unilateral nasal cavity masses with frequent epistaxis, pain or bone erosions should be submitted for histopathological examination.

Key words: Polypoid nasal masses, invasive fungal infections, eosinophilic angiocentric fibrosis.

Article Citation: Khan AA, Asghar AS, Ishaq M, Akhund IA. Polypoid nasal masses; surgical pathology. Professional Med J 2017;24(11):1713-1718.

DOI:10.17957/TPMJ/17.3909
INTRODUCTION

Polypoid nasal masses are commonly encountered in the clinical oto-rhino-laryngology practice. As most of these lesions would be diagnosed ultimately as non-neoplastic nasal polyps, therefore the usual perception developed over the years is not to send the removed nasal polypoid lesions/masses to the pathology laboratory for examination under the microscope. It should be remembered that any localized proliferative or inflammatory process in the nasal tissues will ultimately present as a polypoid mass in the nasal cavity. At times it is impossible clinically to differentiate definitely between neoplastic and non-neoplastic polypoid masses. ${ }^{1}$ The polypoid nasal masses can have diverse underlying etiologies and morphologies, meriting appropriately tailored management and follow-up.

The purpose of this study is firstly to signify the occurrence of unusual nasal polypoid masses by analyzing their histopathological spectrum and to be on the lookout for such entities. Secondly to highlight the fact that nasal polyps should not be taken lightly as they may be representing an underlying ominous pathologic process requiring urgent medical attention. 


\section{MATERIALS AND METHODS}

In this retrospective pathology laboratory based study spanning over a period of five years (from March 2010 to March 2015). All the cases with clinical diagnosis of nasal polyp were retrieved from archives of Charsada Teaching Hospital affiliated with Jinnah Medical College Peshawar. As a departmental policy all the samples are fixed in $10 \%$ formalin. The slides and diagnoses of all the retrieved cases were reviewed and any additional stains etc were performed where necessary. All cases included in the study were with a clinical diagnosis of polypoid nasal lesion with no suspicion for malignancy. Cases included were irrespective of biopsy techniques, gender, age and other demographics.

\section{RESULTS}

On analyzing all the cases of nasal polypoid lesions $(n=155)$ from the year March 2010- March 2015 (Table-I), it was found that large majority of the polyps were non-neoplastic $(n=141,91 \%)$ while the neoplastic lesions comprised the rest $(n=14$, $9 \%$ of all the polypoid lesions). Inflammatory and allergic polyps ( $n=131,92.2 \%$ of non-neoplastic lesions) constituted the most important subcategory of non-neoplastic lesions. Fungal infections ( $n=9,6.4 \%$ of non-neoplastic lesions) constituted the next important subcategory. This was followed by nasal eosinophilic angiocentric fibrosis ( $n=1,0.71 \%$ of non-neoplastic lesions).
The neoplastic lesions $(n=14,9 \%$ of all the polypoid lesions) comprised of benign neoplasms ( $n=10,71.4 \%$ of neoplastic lesions) and malignant neoplasms ( $n=4,28.6 \%$ of neoplastic lesions). The benign masses constituted of hemangioma ( $n=4,28.6 \%$ of neoplastic lesions), angiofibroma ( $n=3,21.4 \%$ of neoplastic lesions), inverted papilloma ( $n=2,14.2 \%$ of neoplastic lesions) and schwannoma ( $n=1,7.1 \%$ of neoplastic lesions). The malignant nasal masses constituted one each ( $n=1,7.1 \%$ of neoplastic lesions) of polypoid extra skeletal Ewingssacoma, plasmacytoma, nonHodgkin lymphoma and renal cell carcinoma.

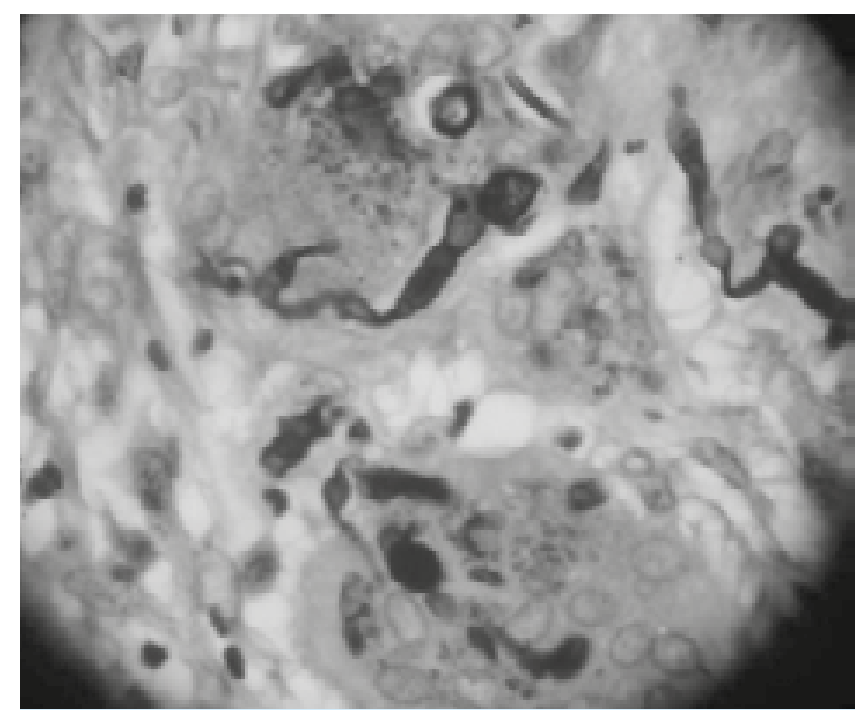

Figure-1. Fungal Rhino-sinusitis: Irregular thick fungal hyphae in multinucleate giant cells. GMS stain, $\mathbf{x} 400$.

\begin{tabular}{|l|c|l|}
\hline \multicolumn{1}{|c|}{ Category } & Number of cases & \multicolumn{1}{c|}{ Percent (\%) } \\
\hline Non-neoplastic & 131 & $92.9 \%$ of non-neoplastic masses \\
\hline Inflammatory+Allergic & 9 & $6.4 \%$ of non-neoplastic masses \\
\hline Fungal & 1 & $0.71 \%$ of non-neoplastic masses \\
\hline Eosinophilicangiocentric fibrosis & & \\
\hline Neoplastic & 4 & $28.6 \%$ of neoplastic masses \\
\hline Benign & 3 & $21.4 \%$ of neoplastic masses \\
\hline Hemangioma & 2 & $14.3 \%$ of neoplastic masses \\
\hline Angiofibroma & 1 & $7.1 \%$ of neoplastic masses \\
\hline Inverted papilloma & 1 & \\
\hline Schwannoma & 1 & $7.1 \%$ of neoplastic masses \\
\hline Malignant & 1 & $7.1 \%$ of neoplastic masses \\
\hline Ewing sarcoma & 1 & $7.1 \%$ of neoplastic masses \\
\hline Plasmacytoma & 155 & \\
\hline Non-Hodgkin Lymphoma & & $7.1 \%$ of neoplastic masses \\
\hline Renal cell carcinoma & Table-I. Number of cases for each category. \\
\hline Total number of cases: & & \\
\hline & &
\end{tabular}




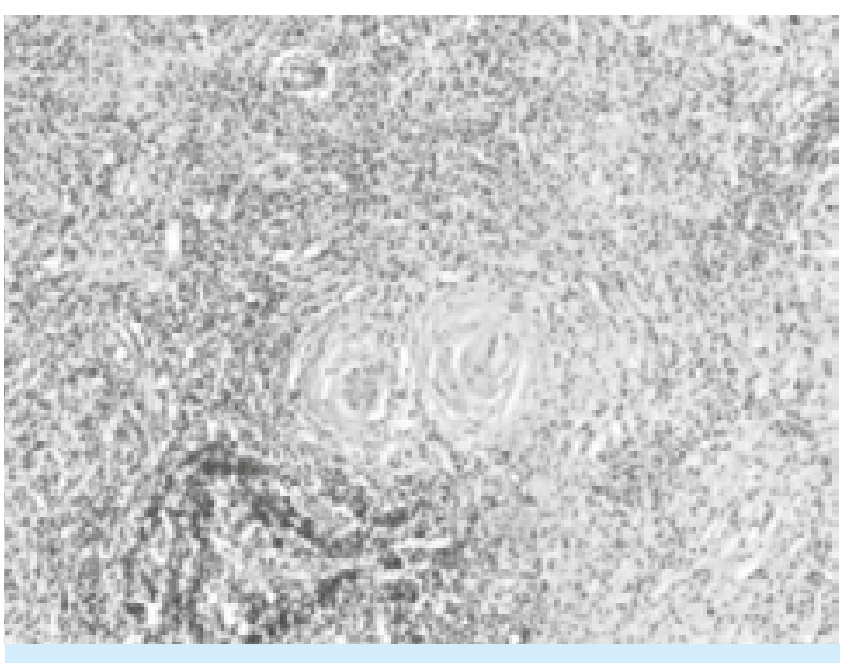

Figure-2. EosinophilicAngiocentric Fibrosis: Perivascular fibrosis and mixed lymphocytic infiltrate with eosinophils. H \& E, x100.

\section{DISCUSSION}

Polypoid nasal masses are commonly encountered in otorhinolaryngology clinics. ${ }^{1,2}$ These masses are inclusive of all the soft tissue proliferations of different etiologies growing into the nasal cavity. The possible etiologies include inflammation, allergy, recurrent infections, cystic fibrosis (rarely), as a complication of mucopolysaccharidosis (Hurler-Scheie syndrome $)^{3}$, but at times the underlying cause cannot be ascertained. ${ }^{4,5}$ The commonest type of polypoid nasal mass is non-neoplastic, therefore most of thesemasses are not sent to the pathology laboratory for histopathological examination. However, rarely a benign or malignant tumor arising from nasal tissue may hang into the nasal cavity closely mimicking a non-neoplastic nasal polyp, thereby apparently obviating the need for histopathological examination, implying missing of few but very important cases of invasive fungal or neoplastic masses. The patients with nasal polypoid nasal masses commonly present with nasal obstruction, anosmia, chronic nasal discharge, recurrent sneezing, recurrent headaches, speech with nasal twang and even epistaxis. ${ }^{4,5}$

Of all the cases of nasal polypoid masses $(n=155)$ reviewed in the pathology laboratory of Charsada Teaching Hospital affiliated with Jinnah Medical

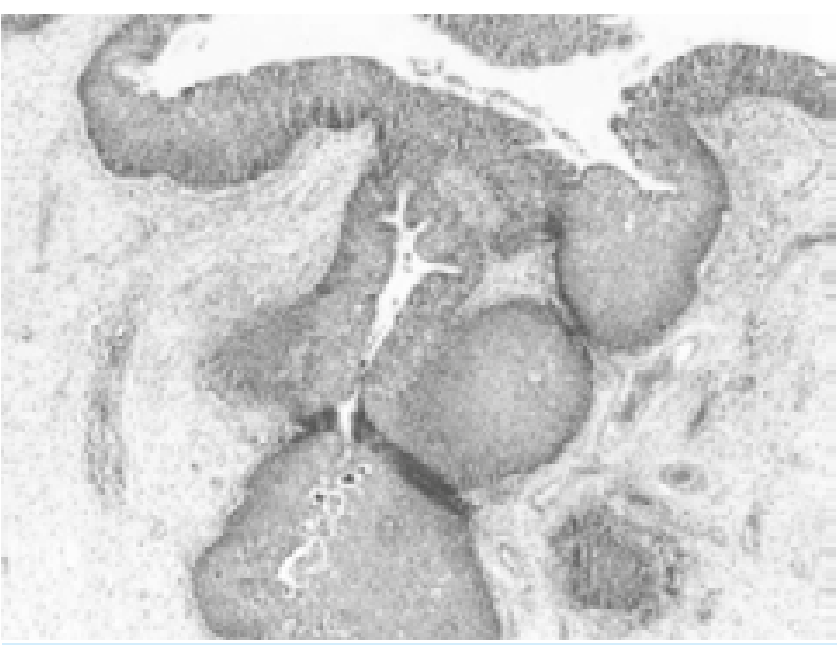

Figure-3. Inverted Papilloma: Endophytic proliferation of non-keratinizing stratified squamous epithelium with scattered mucinous cells covering loose fibrovascular stroma. H \& E, x100.

College Peshawar, most of these nasal polypoid masses (Table-l) belonged to the non-neoplastic category $(n=141,91 \%)$. The inflammatory and allergic polyps representing the maximum number $(n=131,92.9 \%$ of non-neoplastic masses). These inflammatory and allergic nasal polyps were characterized by multiple translucent polypoid fragments composed of edematous stroma infiltrated by lymphocytes, plasma cells, eosinophils and mast cells. There was accompanying mucinous glandular hyperplasia, with increased vascularity towards the base. In polyps of allergic etiology the epithelial basement membrane was thick with abundant eosinophils and needle-like Charcot-Leyden crystals in the edematous stroma. This preponderance of non-neoplastic polyps is in corroboration with various studies, where the reported proportion of such polyps varies from $50.7 \%$ to $73.6 \% .^{1,6}$ The increased number of non-neoplastic masses in our study can be attributed to increased air pollutants, allergies and late reporting to the hospitals to seek medical advice. However, the proportion of inflammatory and allergic nasal polyps inanother conducted study by Garg et al is almost the same $(89.5 \%)$ as in this study. ${ }^{6}$

Nasal polyps with fungal infections $(n=9,6.4 \%$ of non-neoplastic masses) were responsible for the second most common non-neoplastic mass. 
These polypoid fragments were dark, opaque and covered by muddy and thick mucus. They were densely infiltrated by lymphocytes, plasma cells, eosinophils, histiocytes and multinucleated giant cells. Some of the cystically dilated mucous acini contained fungal hyphae (Figure-1) without stromal invasion (non-invasive). While in a few cases the fungal hyphae were seen invading the stroma (invasive form) and were being surrounded by chronic inflammatory reaction and multinucleate giant cells with ingested fragments of fungal hyphae. Invasive fungal infection needs to be reported as soon as possible, as urgent management is required in order to prevent spread to the nearby brain. ${ }^{7}$ A similar figure of $9.2 \%$ of the non-neoplastic masses has been reported by Kumariet al. ${ }^{8}$

Eosinophilic angiocentric fibrosis ( $n=1,6.4 \%$ of all non-neoplastic lesions) was the next frequent non-neoplastic lesion encountered (Figure-2), represented by a single case having nasal obstruction with no response to local medication. On examination there was a firm mass in the left nasal cavity. On radiological examination there was erosion of the medial orbital plate, it was morphologically characterized by perivascular concentric fibrosis and eosinophil rich lymphoplasmacytic infiltrate. Its exact etiology is not known, however an IgG4 associated disorder has been proposed as the most probable etiology in a study showing increased serum IgG4 levels and increased number of IgG4 positive plasma cells in such lesions. . $^{9,10,11,12}$

Amongst the neoplastic polypoid masses $(n=14$, $9 \%$ of all polypoid lesions), benign masses ( $n=10,71.4 \%$ of all the neoplastic lesions) were more common than the malignant neoplasms ( $n=4,28.6 \%$ of all the neoplastic lesions). This distribution of benign and malignant nasal polypoid masses is in keeping with that reported by Dasgupta et al (benign: $75.9 \%$, malignant: $24.1 \%){ }^{1}$

Hemangioma $(n=4,28.6 \%$ of all neoplastic lesions) was the most common benign mass, presenting with discharge and repeated epistaxis. Microscopically there was lobular capillary architecture with surface epithelial ulceration; it has been reported as $18.2 \%$ of benign neoplasms by Garget al. ${ }^{6}$ Angiofibroma ( $n=3$, $21.4 \%$ of all neoplastic lesions) was the next common benign neoplastic mass represented by three patients, the common presentation was by recurrent epistaxis from a shiny white colored mass. Histopathologically it was characterized by thin walled dilated angulated or irregular shaped blood vessels set in fibrocollagenous stroma containing plump spindle to stellate shaped fibroblasts, reported as occurring in $23.2 \%$ of benign neoplasms by Dasguptaet al. ${ }^{1}$ Inverted papilloma ( $n=2,14.3 \%$ of all neoplastic lesions) was the third most common benign neoplastic mass (Figure-3), reported as 3.9\% by Dasgupta et al. ${ }^{1}$ These cases presented with progressive nasal obstruction and discharge. Nasal examination showed tan-pink, polypoid growth arising from the lateral nasal wall. Histopathological examination revealed endophytic proliferation of non-keratinizing markedly acanthotic stratified squamous epithelium with scattered mucinous cells. The stroma was edematous and mildly infiltrated by chronic inflammatory cells. One of the patients presented three years later with recurrence that was treated successfully by surgery alone. Schwannoma $(n=1,7.1 \%$ of all neoplastic lesions) was the fourth most common benign neoplasm. It was composed of dense and rarified areas with scattered blood vessels having hyaline walls, surface ulceration and focal surface acute inflammatory exudates. These benign neoplasms have almost the same distribution as reported in other studies like that by Garget et al. ${ }^{6}$

The malignant neoplastic category $(n=4,28.6 \%$ of all neoplastic lesions) comprised one each $(n=1$, $7.1 \%$ of all neoplastic lesions) of Ewing's sarcoma, plasmacytoma, non-Hodgkins lymphoma and metastatic renal cell carcinoma. Ewing's sarcoma presented with left sided nodular polypoid mass in an eight years old girl causing nasal obstruction and discharge, the biopsy fragments were composed of lobular proliferation of malignant cells having scanty cytoplasm, round to oval nuclei with coarse granular chromatin admixed with cells having nuclei with smudged chromatin. There was conspicuous apoptosis and necrosis. 
A provisional diagnosis of small blue cell tumor was rendered and the final diagnosis from the reference laboratory was extra-skeletal Ewing's sarcoma. The patient came back two years later after having non-compliant medication with a more aggressive and extensive tumor causing patient's death due to involvement of brain. The non-Hodgkin lymphoma presented in a 75 years old male as progressive nasal obstruction and headache with occasional nose bleed for five months. A left sided nasal polypoid mass was discovered with progressive increase in the polyp size accompanied by unilateral swelling of the face and erosion of the midline structures. The biopsy fragments showed sheets of small lymphoid cells. A provisional diagnosis of nonHodgkin lymphoma was rendered and the final diagnosis from the reference laboratory was $T$ cell lymphoma. Plasmacytoma presented in a 80 years old male with right sided nasal mass for many months with occasional epistaxis and frequent difficulty in breathing through nose. The respected mass was composed of sheets of mature and immature plasma cells showing occasional bi-nucleation and was covered by respiratory type of epithelium, features suggestive of plasmacytoma. Metastatic renal cell carcinoma presented in a 62 years old female with epistaxis and nasal obstruction for two weeks. On examination there was an easy to bleed red polypoid mass in the nasal cavity. There was history of radical nephrectomy for renal cell carcinomasix years back. Histopathological examination of the polypoid mass showed clusters of mildly pleomorphic clear cells separated by thinned walled capillaries with focal areas of hemorrhages, representing metastatic renal cell carcinoma. The reported incidence of malignant masses in the nasal cavity has been variable in different published studies. Squamous cell carcinoma has been reported as the most common malignant tumor in different studies. ${ }^{1,2,7,13,14}$ whereas it is not encountered in this study; the probable reasons being firstly, this study included 155 patients whereas in other published studies the patient number was in excess of two hundred, secondly, all the patients in this study were Muslims who have to clean their nasal cavity with water at least five times a day before each mandatory salah (prayers).

In conclusion, it is to be remembered that all nasal cavity masses are not inflammatory or benign, the histopathologist should be aware that all the above mentioned entities and many more can be encountered in the nasal cavities. Important diagnostic categories requiring urgent attention for management include polyps with invasive fungal infection, inverted papillomas and all the malignant cases. Therefore, all unilateral nasal cavity masses with frequent epistaxis, pain or bone erosions should be submitted for histopathological examination.

Copyright@ 15 Sep, 2017.

\section{REFERENCES}

1. Dasgupta A, Ghosh RN, Mukherjee C. Nasal Polyps - Histopathological Spectrum. Indian Journal of Otolaryngology and Head \& Neck Surgery. Vol. 49, No. 1, Jan-March, 1997.

2. Khan N, Zafar U, Afroz N, Ahmad S, Hassan S. Masses of Nasal Cavity, Paranasal Sinuses and Nasopharynx: A Clinicopathological Study. Indian Journal of Otolaryngology and Head \& Neck Surgery. Vol. 58, No. 3, July-September, 2006.

3. Juan R. Respiratory Tract: In: Rosai and Ackerman's Surgical Pathology (10 ${ }^{\text {th }}$ edition). Elsevier: 2011: 292308.

4. Newton JR, Ah-See KW. A Review of Nasal Polyposis. Therapeutics and Clinical Risk Management. 2008 April;4(2):507-12.

5. Bruce MW. Nasal Cavity, Paranasal Sinuses, and Nasopharynx. In: Weidner N, Cote RJ, Suster S, Weis LM. Modern Surgical Pathology; Saunders; ( $2^{\text {nd }}$ edition) 2009. 143-196.

6. Garg D, Mathur K. Clinico-pathological Study of Space Occupying Lesions of Nasal Cavity, Para nasal Sinuses and Nasopharynx. Journal of Clinical and Diagnostic Research. 2014 November, Vol. 8(11): FC04-FC07.

7. Johnson MM. Ear, Nose, and Throat Infections. In: Kradin RL. Diagnostic Pathology of Infectious Disease; Saunders; ( $1^{\text {st }}$ edition) 2010. 107-112.

8. Kumari K, Mahadeva KC. Polypoidal Lesions in the Nasal Cavity. Journal of Clinical Diagnostic Research. 2013 June, Vol. 7(6): 1040-1042.

9. Deshpande V, Khosroshahi A, Nielsen GP, Hamilos 
$\mathrm{DL}$, Stone $\mathrm{JH}$. Eosinophilicangiocentric fibrosis is a form of IgG4-related systemic disease. American Journal of Surgical Pathology. 2011 May; 35(5):701-6.

10. Onder S, Sungur A. Eosinophilicangiocentric fibrosis: an unusual entity of the sinonasal tract. Arch Pathol Lab Med. 2004 Jan; 128(1):90-1.

11. Fang $\mathrm{CH}$, Mady LJ, Mirani NM, Baredes S, Eloy JA. Sinonasaleosinophilicangiocentric fibrosis: a systematic review. International Forum of Allergy and Rhinology. 2014 Sep; 4(9):745-52.

12. Bruce MW. Diseases of the Nasal Cavity, Paranasal
Sinuses, and Nasopharynx. In: Leon Barnes, Surgical Pathology of the head and neck, Informa healthcare; (Third Edition) 2009. 363-402.

13. Bruce MW. The Nose, Paranasal Sinuses, and Naopharynx. In: Stacey EM. Sternberg's Diagnostic Surgical Pathology; Lippincott Williams \& Wilkins; (5 $5^{\text {th }}$ edition) 2010. 865-892.

14. Gustave LD.Nonsquamous Lesions of the Nasal Cavity, Paranasal Sinuses, and Nasopharynx. In: GneppR. Diagnostic Surgical Pathology of The Head and Neck; Saunders; (2nd edition) 2009. 112-117.

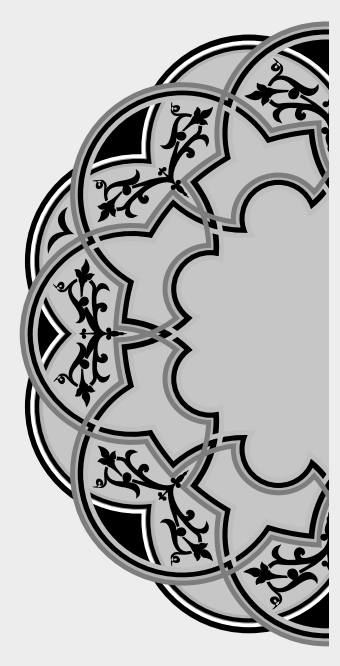

\title{
"The time is always right to do what is right."
}

\author{
Martin Luther King Jr.
}

AUTHORSHIP AND CONTRIBUTION DECLARATION

\begin{tabular}{|c|c|c|c|}
\hline Sr. \# & Author-s Full Name & Contribution to the paper & Author $=\mathbf{s}$ Signature \\
\hline 1 & Amjad Ali Khan & $\begin{array}{l}\text { Concept and design, Data } \\
\text { collection, Analysis and } \\
\text { interpretation, Manuscript } \\
\text { drafting, Manuscript revision. }\end{array}$ & \\
\hline 2 & Abdul Shaheed Asghar & $\begin{array}{l}\text { Concept and design, Data } \\
\text { analysis and interpretation, } \\
\text { Manuscript drafting, } \\
\text { Manuscript revision. }\end{array}$ & \\
\hline 3 & Muhammad Ishaq & $\begin{array}{l}\text { Concept and desidn, Data } \\
\text { interpretation, Manuscript } \\
\text { revision. }\end{array}$ & \\
\hline 4 & Israr Ahmed Akhund & $\begin{array}{l}\text { Data collection and analysis, } \\
\text { Manuscript drafting, } \\
\text { Manuscript revision. }\end{array}$ & \\
\hline
\end{tabular}

